\title{
KINEMATICS OF ORION ASSOCIATION MEMBERS
}

\author{
R.L.SMART \\ Space Telescope and Science Institute
}

We discuss the determination of the expansion of the Orion association. Previous estimates of expansion in associations have employed simplified linear approximations which only required the observation of proper motions (cf. Lesh,J.R.,ApJ, 152, 905, 1968). We have used proper motions obtained by Smart (PhD Thesis, Univ. of Florida, 1993) with previously obtained membership criteria to investigate this hypothesised expansion without conclusive results.

In future work we intend to combine these proper motions with new radial velocity observations and spectroscopic distances from the literature to find the expansion using the rigorous formulation (Eichhorn, H. K., Kinematic Astrometry, in prep., 1994):

$$
\left(\begin{array}{c}
\sigma r-\dot{r} \\
r \dot{\alpha} \cos \delta \\
r \dot{\delta}
\end{array}\right)=\sigma r_{o}\left(\begin{array}{c}
\cos \delta \cos \delta_{o} \cos \left(\alpha-\alpha_{o}\right)+\sin \delta \sin \delta_{o} \\
\cos \delta_{o} \sin \left(\alpha-\alpha_{o}\right) \\
\sin \delta \cos \delta_{o} \cos \left(\alpha-\alpha_{o}\right)-\cos \delta \sin \delta_{o}
\end{array}\right) \text {. }
$$

Where the parameters $\alpha_{o}, \delta_{o}, r_{o}$ and $\sigma$ are the positions, distance and reciprocal of the expansion age of the 'original' explosion respectively. The observations are $\alpha, \delta, r, \dot{r}$ and $\dot{\alpha}, \dot{\delta}$ are the positions, distance, radial velocity and proper motions of the individual stars. The parameters of the expansion can be solved for using an iterative least squares technique.

Since this region covers over 30 square degrees this study will require the use of sophisticated widefield techniques. For the astrometric reductions we have used the overlapping plate technique on photographic plates from three epochs. For the radial velocity observations we plan to use the FLAIR camera of the UK Schmidt. The projected error of the radial velocities $(\mathrm{rms}=5 \mathrm{~km} / \mathrm{s})$ matches the estimated error of the proper motions $(\mathrm{rms}=2 \mathrm{mas} / \mathrm{yr})$ at the nominal distance of Orion $(450 \mathrm{pc})$. These observations can be used to restrict theories of stellar association formation, runaway stars, evolutionary ages, pre-stellar gas dynamics and galactic kinematics. 